\title{
MULTIPLE COMPARISONS IN CARCINOGENESIS STUDY WITH RIGHT-CENSORED SURVIVAL DATA
}

\author{
YUH-ING CHEN* \\ Institute of Statistics, National Central University, Chung-Li, Taiwan 320, R.O.C.
}

\begin{abstract}
SUMMARY
This paper considers the practical problem in animal carcinogenesis experiments where several treatment groups are compared with a control group in a one-way layout and the observed survival data are subject to random right-censorship. Proposed herein are multiple testing procedures based on two-sample weighted logrank statistics, each comparing an individual treatment with the control, for determining which treatments are more effective than the control. The associated $p$-value of claiming a certain treatment is more effective than the control is also discussed. A test-based confidence set for the scale changes between each treatment and the control is then obtained. The comparative results of a Monte Carlo error rate and power study for small sample sizes are presented. Finally, a numerical example involving renal carcinoma in mice demonstrates the feasibility of the proposed multiple testing procedures and test-based confidence set. Copyright (C) 2000 John Wiley \& Sons, Ltd.
\end{abstract}

\section{INTRODUCTION}

Statistical procedures for comparing several treatments with a control have been extensively discussed when data are completely observed in a one-way layout (see, for example, Hochberg and Tamhane ${ }^{1}$ ). In animal carcinogenesis experiments, different therapies (treatments) are usually compared with a standard therapy or placebo (control) to evaluate the effect of the treatments on the prolongation of the survival time of animals with a certain established carcinoma. Of particular interest are those treatments in which effects exceed that of the control. However, in these studies, survival data are frequently subject to random right-censorship, since the study may be terminated at a pre-assigned time owing to time limitation, or the death may be attributed to a competing risk which is not of interest. Therefore, statistical procedures are needed for the many-to-one comparisons with randomly right-censored survival data.

Multiple testing procedures for the many-to-one comparisons problem with right-censored data have been recently developed. For instance, Chakraborti and Desu ${ }^{2}$ used Slepian's ${ }^{3}$ inequality to suggest a conservative multiple testing procedure based on Gehan's ${ }^{4}$ twosample statistics each comparing an individual treatment with the control. Chen ${ }^{5}$ proposed a

* Correspondence to: Yuh-Ing Chen, Institute of Statistics, National Central University, Chung-Li, Taiwan 320, R.O.C. E-mail: ychen@stat.ncu.edu.tw

Contract/grant sponsor: National Science Council of Taiwan

Contract/grant number: NSC 86-2115-M-008-018

CCC 0277-6715/2000/030353-15\$17.50

Copyright (C) 2000 John Wiley \& Sons, Ltd.

Received April 1998

Accepted March 1999 
generalization of Steel's ${ }^{6}$ test on the basis of the maximum of Gehan's ${ }^{4}$ statistics for the special case of equal censoring. However, the logrank statistic (Mantel ${ }^{7}$ ) is probably the most commonly used two-sample statistic and Gehan's statistic is a member of the general class of weighted logrank statistics (see, for example, Fleming and Harrington ${ }^{8}$ ). Chen ${ }^{9}$ further extended the Chakraborti-Desu testing procedure on the basis of the two-sample weighted logrank statistics.

In this paper we consider an alternative generalization of Steel's test for the many-to-one comparisons based on the two-sample weighted logrank statistics each comparing an individual treatment with the control. Based on the generalized Steel's test, a confidence set for the ratios of scale parameters of each treatment and the control in a scale-change model is obtained (Wei and Gail ${ }^{10}$ ). In addition, a closed testing procedure (Marcus et al. ${ }^{11}$ ) is suggested in which the generalized Steel's test is modified. Three special weighted logrank statistics under consideration are Gehan's statistic, the logrank statistic and the Peto-Prentice statistic (Peto and Peto ${ }^{12}$ and Prentice $^{13}$ ). Moreover, for the conclusion that a certain treatment is more effective than the control reached by the proposed testing procedure, the associated $p$-value defined to be the smallest overall significance level for obtaining the conclusion is discussed. Comparative results of a Monte Carlo study investigation demonstrate the relative error rate and power performances of the proposed testing procedures for small sample sizes. Finally, the use of these testing procedures is illustrated with the numerical example involving renal carcinoma in mice described in Section 2.

\section{A RENAL CARCINOMA EXAMPLE}

Interleukin 2 (IL-2) and interleukin 12 (IL-12) are potent immunoregulatory cytokines that exhibit anti-tumour activity (see, for example, Gately ${ }^{14}$ and Maas et al. ${ }^{15}$ ). Preliminary evidence further suggests that combined administration of IL-12 and IL-2 may yield greater anti-tumour activity than that observed with either agent alone (see, for instance, Rossi et al. ${ }^{16}$ ). To evaluate the ability of IL-2, IL-12 and combination regimens of IL-2 and IL-12 to induce regression of established primary and metastatic murine renal carcinoma (Renca) tumours, Wigginton et al. ${ }^{17}$ conducted an animal experiment. Forty BALB/c mice administered an internal injection of $1 \times 10^{5}$ Renca cells and developing subcutaneous primary Renca tumours were divided into four groups to receive no treatment (control), IL-2 (300 000 IU given twice daily one day per week) alone, IL-12 (0.5 $\mu \mathrm{g}$ given on a daily basis) alone, or IL-12 in combination with IL-2. The measurement of record for each group was the survival time after tumour injection. However, the mice survived and experienced tumour regression at the end of the study, yielding censored data.

In this study, the anti-tumour activity of IL-12 in combination with IL-2, IL-2 or IL-12 alone on regression of the established Renca tumours needs to be evaluated. Whether treatment with IL-12 plus IL-2 displays greater anti-tumour activity against the established Renca tumours than either IL-12 or IL-2 alone also needs to be investigated. To this end, testing procedures preserving a correct overall significance level are needed for determining simultaneously which of IL-2 alone, IL-12 alone, and the combined administration of IL-12 and IL-2, comparing to the control, can yield anti-tumour activity against the Renca tumours. Testing procedures holding a correct overall significance level are also required for simultaneously comparing the combined IL-12 and IL-2 with IL-12 alone and IL-2 alone. Note that the involving multiple comparisons are, in fact, corresponding to the many-to-one comparisons. 


\section{PROPOSED TESTING PROCEDURES}

Let $S_{i}$ be the survival function of the $i$ th group, $i=0,1, \ldots, m$. Suppose that the zero population $(i=0)$ is the control and the other $m$ populations are treatments. In such a setting with rightcensored data, we only observe the bivariate vectors $\left(X_{i u}, \delta_{i u}\right), u=1, \ldots, n_{i}, i=0,1, \ldots, m$, where $X_{i u}$ is the minimum of the survival time and the associated censoring time, and $\delta_{i u}$ is the indicator of censorship, which is one if the observation is not censored, and zero otherwise. In this paper, specifically, we are concerned with the many-to-one multiple comparisons problem of determining the treatments which are more effective than the control; that is, $S_{i}>S_{0}, i=1, \ldots, m$, when survival data are subject to random right-censorship.

Let $T_{1}<\cdots<T_{L}$ denote the ordered observed distinct death times in the sample formed by combining the $i$ th and control groups. Let $d_{u k}$ and $Y_{u k}, k=1, \ldots, L, u=0, i$, denote the number of observed deaths and number at risk, respectively, in sample $u$ at time $T_{k}$. Set $d_{+k}=d_{0 k}+d_{i k}$ and $Y_{+k}=Y_{0 k}+Y_{i k}$. Note that, given $d_{+k}, Y_{0 k}$ and $Y_{i k}$, the conditional distribution of $d_{0 k}$ is the hypergeometric distribution with mean $e_{0 k}=d_{+k} Y_{0 k} / Y_{+k}$ and variance $v_{k}=d_{+k} Y_{0 k} Y_{i k}$ $\left(Y_{+k}-d_{+k}\right) /\left\{\left(Y_{+k}\right)^{2}\left(Y_{+k}-1\right)\right\}$. For some appropriate weight functions $W_{i}\left(T_{k}\right)$, let

$$
U_{0 i}=\sum_{k=1}^{L} W_{i}\left(T_{k}\right)\left(d_{0 k}-e_{0 k}\right)
$$

and

$$
S_{i i}=\sum_{k=1}^{L}\left\{W_{i}\left(T_{k}\right)\right\}^{2} v_{k} .
$$

Then, under significance level $\alpha$, the two-sample weighted logrank tests conclude $S_{i}>S_{0}$, if

$$
U_{i}=U_{0 i} / \sqrt{ } s_{i i} \geqslant z(\alpha)
$$

where $z(\alpha)$ is the upper $\alpha$ th percentile of the standard normal distribution. Note that taking $W_{i}\left(T_{k}\right)=Y_{+_{k}} /\left(n_{0}+n_{i}\right)$ to be the proportion at risk at time $T_{k}$ produces Gehan's statistic, taking $W_{i}\left(T_{k}\right)=1$ yields the logrank statistic, and setting $W_{i}\left(T_{k}\right)$ to be the Kaplan-Meier ${ }^{18}$ survival estimate based on the pooled samples from the $i$ th and the control groups gives the Peto-Prentice statistic. These three special cases of the weighted logrank statistics are of general interest, since they approximate many practical applications and are available in most statistical packages.

To obtain a generalized Steel's many-to-one comparisons procedure which controls the overall significance level or experimentwise error rate (probability of erroneously declaring at least one treatment more effective than the control), we need to find the percentiles of the distribution of $\max \left(U_{1}, U_{2}, \ldots, U_{m}\right)$ under the null hypothesis $H_{0}:\left(S_{i}=S_{0}, i=1,2, \ldots, m\right)$. Note that, as specified in the Appendix, the asymptotic null distribution of $\left(U_{1}, U_{2}, \ldots, U_{m}\right)$ is an $m$-variate normal with mean zero and covariance matrix $\Sigma=\left(\rho_{i j}\right)$, which can be consistently estimated by $\mathbf{S}$. Let $\left(Z_{1}, Z_{2}, \ldots, Z_{m}\right)$ be an $m$-variate normal vector with mean zero and covariance matrix $\mathbf{S}$. As a generalization of Steel's testing procedure, we suggest

$$
\text { SMAX: claim } S_{i}>S_{0} \text {, if } U_{i} \geqslant z \max (m, \alpha), \text { for } i=1,2, \ldots, m
$$


where $z \max (m, \alpha)$ is the upper $\alpha$ th percentile of the distribution of $\max \left(Z_{1}, Z_{2}, \ldots, Z_{m}\right)$. Obviously, the experimentwise error rate for the procedure (3) is approximately controlled, since

$$
\begin{aligned}
\alpha & \approx P\left\{\max \left(U_{1}, U_{2}, \ldots, U_{m}\right) \geqslant z \max (m, \alpha) \mid H_{0}\right\} \\
& =P\left\{U_{i} \geqslant z \max (m, \alpha) \text { for at least one } i=1,2, \ldots, m \mid H_{0}\right\} .
\end{aligned}
$$

Note that, for any $z$, the probability $P\left\{\max \left(Z_{1}, Z_{2}, \ldots, Z_{m}\right) \leqslant z\right\}$ can be computed using a program for calculating multivariate normal probabilities $\left(\right.$ Schervish $\left.{ }^{19}\right)$. Therefore, the critical value $z \max (m, \alpha)$ can be found such that $P\left\{\max \left(Z_{1}, Z_{2}, \ldots, Z_{m}\right) \geqslant z \max (m, \alpha)\right\}=\alpha$. However, when the $m+1$ groups under consideration have the same censoring distribution, for the three weight functions considered herein, the result in $\mathrm{Chen}^{9}$ implies that $\rho_{i j}=1$, if $i=j$, and $\sqrt{ }\left\{n_{i} n_{j} /\left(n_{0}+n_{i}\right)\left(n_{0}+n_{j}\right)\right\}$ otherwise. Moreover, for the particular case of treatment-balanced design with $n_{0}=c$ and $n_{1}=\cdots=n_{k}=n$, we observe $\rho_{i j}=n /(n+c)$, if $i \neq j$. Therefore, when the assumption of equal censoring is tenable, we recommend using the critical value corresponding to the known covariance matrix $\Sigma=\left(\rho_{i j}\right)$ which can be computed by using the program in Schervish $^{19}$ or found in Gupta ${ }^{20}$ for the treatment-balanced design. Also note the conservative procedure based on Slepian's inequality proposed by Chen: ${ }^{9}$

CHEN: claims $S_{i}>S_{0}$, if $U_{i} \geqslant z(b), i=1,2, \ldots, m$

where $b=1-(1-\alpha)^{1 / m}$.

Suppose that the survival times in the $m+1$ groups satisfy the scale-change model; that is, $F_{i}(x)=F\left(x / \theta_{i}\right)$, with $F_{i}=1-S_{i}, i=0,1, \ldots, m$. Set $\gamma_{i}=\theta_{i} / \theta_{0}$ to be the ratios of the scale parameters of the $i$ th treatment group and the control. Let $\mathbf{X}_{i}=\left(X_{i 1}, X_{i 2}, \ldots, X_{i n_{i}}\right)$ and $\Delta_{i}=\left(\delta_{i 1}, \delta_{i 2}, \ldots, \delta_{i n_{i}}\right), i=0,1, \ldots, m$. Denote the $U_{0 i}$ in (1) and $s_{i i}$ in (2) as $h\left(\mathbf{X}_{0}, \mathbf{X}_{i}, \Delta_{0}, \Delta_{i}\right)$ and $V\left(\mathbf{X}_{0}, \mathbf{X}_{i}, \Delta_{0}, \Delta_{i}\right)$, respectively. Therefore, by applying the results in Wei and Gail, ${ }^{10}$ an approximate $(1-\alpha) \times 100$ per cent confidence set for $\gamma=\left(\gamma_{1}, \gamma_{2}, \ldots, \gamma_{m}\right)$ based on the test in (3) is given by

$$
C(\gamma)=\left\{\left(\gamma_{1}, \gamma_{2}, \ldots, \gamma_{m}\right): \gamma_{i} \geqslant \gamma_{i}, \quad i=1,2, \ldots, m\right\}
$$

where $\gamma_{i}$ is the smallest value of $a$ such that

$$
h\left(\mathbf{X}_{0}, \mathbf{X}_{i} / a, \Delta_{0}, \Delta_{i}\right) \leqslant z \max (m, \alpha) \sqrt{ }\left\{V\left(\mathbf{X}_{0}, \mathbf{X}_{i} / a, \Delta_{0}, \Delta_{i}\right)\right\} .
$$

If only a significance testing procedure is of interest, a stepwise comparison is then required which is generally more powerful than a single step procedure. For the problem considered herein, the class of null hypotheses $H=\left\{H_{0}(P)\right\}$, where $H_{0}(P):\left(S_{i}=S_{0}\right.$ for $i$ in $\left.P\right)$ and $P$ is any subset of $\{1,2, \ldots, k\}$, is closed under intersection in the sense that both $H_{0}(P)$ and $H_{0}(Q)$ in $H$ implies the intersection of $H_{0}(P)$ and $H_{0}(Q)$ also in $H$. Therefore, to determine which treatments are more effective than the control with right-censored survival data, a closed testing procedure (Marcus et al. ${ }^{11}$ ) can be constructed which includes separate $\alpha$-level tests of individual $H_{0}(P)$ applied in a step-down manner. Let $U_{(1)}<U_{(2)}<\cdots<U_{(k)}$ be the ordered $U_{i}$ 's and let $d_{i}$ be the antirank of $i$, that is, $U_{(i)}=U_{d_{i}}$. Denote by $z \max ((i), \alpha)$ the upper $\alpha$ th percentile of the distribution of $\max \left(Z_{d_{1}}, Z_{d_{2}}, \ldots, Z_{d_{i}}\right)$. Consequently, a closed testing procedure is obtained:

$$
\text { CLOSE: claims } \left.S_{d_{i}}>S_{0} \text { if } U_{(i)} \geqslant z \max (i), \alpha\right)
$$


provided that $\left.\left.U_{(j)} \geqslant z \max (j)\right), \alpha\right)$ for $j>i$. This procedure continues until $U_{(k)}<z \max ((k), \alpha)$ for some $k=1,2, \ldots, m$. Finally, we declare at an approximate experimentwise error rate $\alpha$, that only the treatment labelled by $d_{k+1}, \ldots, d_{m}$ are more effective than the control.

Note that the $p$-value of a single test is the smallest significance level which leads to a rejection of the null hypothesis. For the many-to-one comparisons procedure with the conclusion that a certain treatments are better than the control, the associated $p$-value is then defined to be the smallest experimentwise error rate at which the testing procedure reaches the conclusion. Let $u_{(1)}<u_{(2)}<\cdots<u_{(m)}$ be the observed values of $U_{(1)}<U_{(2)}<\cdots<U_{(m)}$. Recall that $u(i)=u_{d_{i}}$, $i=1, \ldots, m$. The $p$-value of the generalized Steel's procedure in (3) with the conclusion that the treatments labelled by $d_{k+1}, \ldots, d_{m}(k<m)$ are more effective than the control is then given by $\left.P\left\{\max \left(U_{1}, U_{2}, \ldots, U_{m}\right) \geqslant u_{d_{k+1}}\right\} \mid H_{0}\right\}$ which can be approximated by

$$
p\left(\operatorname{SMAX} ; d_{k+1}, \ldots, d_{m}\right)=1-P\left\{Z_{1} \leqslant u_{d_{k+1}}, \ldots, Z_{m} \leqslant u_{d_{k+1}}\right\}
$$

where the vector $\left(Z_{1}, Z_{2}, \ldots, Z_{m}\right)$ is, again an $m$-variate normal vector with mean zero and covariance matrix $\mathbf{S}$. Following the results in Dunnett and Tamhane, ${ }^{21}$ let

$$
p^{\prime}\left(d_{i}\right)=1-P\left\{Z_{d_{1}} \leqslant u_{d_{i}}, \ldots, Z_{d_{i}} \leqslant u_{d_{i}}\right\}, \quad i=1,2, \ldots, m .
$$

Then, the approximate $p$-value of the closed test in (6) with the conclusion that the treatments labelled by $d_{k+1}, \ldots, d_{m}$ are more effective than the control is obtained as

$$
p\left(\text { CLOSE; } d_{k+1}, \ldots, d_{m}\right)=\max \left\{p^{\prime}\left(d_{k+1}\right), p^{\prime}\left(d_{k+2}\right), \ldots, p^{\prime}\left(d_{m}\right)\right\} .
$$

Also note that procedures in (3) and (6) have the same experimentwise error rate and experimentwise power (probability of correctly detecting at least one treatment which is better than the control). Meanwhile, since $z \max ((i), \alpha)$ is increasing in $i$, the closed testing procedure in (6) would have a greater comparisonwise power (probability of correctly declaring all treatments which are better than the control) than does the generalized Steel's procedure in (3). However, the closed testing procedure, as a stepwise procedure, cannot be used for constructing a confidence set for the ratios of scale parameters in the scale-change model. Furthermore, procedures (3)-(6) are allowed to employ two-sample weighted logrank statistics with different weight functions.

All the procedures mentioned above are appropriate for the situation where the treatments are expected to be at least as good as the control (right-sided comparisons). However, when it is expected that the control is at least as good as the treatments (left-sided comparisons) or there is at least one treatment which is different from the control (two-sided comparisons), the procedures in (3)-(6) should be modified as stated in the Appendix.

\section{MONTE CARLO STUDY}

\subsection{Discussion of study}

A Monte Carlo study was performed to examine the relative level and power performances of the generalized Steel's testing procedure SMAX in (3), the conservative test CHEN in (4), and the closed testing procedure CLOSE in (6) for comparing several treatments with a control when observations are subject to random right-censorship. For simplicity, we only considered procedures based on two-sample weighted logrank statistics with the same type of weight functions. The level performances of these tests were evaluated by the experimentwise error rate and their power performances were assessed by both the experimentwise and comparisonwise powers. 
In this study, we considered $k=3$ treatments with sample sizes $n_{0}=c, n_{1}=\cdots=n_{3}=n$. We employed $(c, n)=(10,10),(20,20),(30,30)$ and $(30,20)$ in the level study, and $(c, n)=(20,20)$ and $(30,20)$ in the power study. Exponential and log-normal distributions were considered as survival time distributions and the uniform distribution over $(0, R)$ was used as the censoring distribution. Appropriate uniform, normal and exponential variates were generated by using the IMSL routines RNUN, RNNOR and RNEXP, respectively. The necessary log-normal variates were then given by the exponential of the normal variates. In the level study, the standard exponential distribution and the log-normal distribution with zero normal mean and normal standard deviation $\sigma=1 / 2$ were considered. In the power study, we used exponential distributions with various values of scale parameters $\theta_{i}$ 's and log-normal distributions with normal standard deviation $\sigma=1 / 2$ but different values of normal means $\theta_{i}$ 's. Note that the exponential distribution represents the proportional hazards model and the log-normal distribution corresponds to location shifts in log survival times. In fact, the log-normal distributions considered in the power study have different hazards at early times. Various values of $R$ which correspond to the probability of censorship (the probability that survival time is greater than the censoring time), $p$, as $0 \cdot 10$ and 0.30 were considered in the level study, the corresponding uniform distributions were then employed as censoring distributions in the power study. For example, when survival time distribution is the standard exponential and $p=0 \cdot 1, R=9 \cdot 901$. For the log-normal distribution with zero normal mean and normal standard deviation $\sigma=1 / 2, R=3.756$ corresponds to $p=0 \cdot 3$. Note that the censoring probabilities were fixed for each population in the level study, while they may be different for the populations involved in the power study due to different life time distributions.

For each of these settings, 5000 replications were used to obtain the estimated experimentwise error rates or both the experimentwise and comparisonwise powers under the nominal level $\alpha=0.05$. Therefore, the maximum standard error for the estimate is around 0.007 $(\approx \sqrt{ }\{(0 \cdot 5(0 \cdot 5) / 5000\})$. In fact, under the nominal level $\alpha=0 \cdot 05$, the standard error for the estimated error rate is about $0.003(\approx \sqrt{ }(0.05)(0.95) / 5000\})$. We then indicate, by $+(-)$ signs, whenever the estimated error rate is two or more standard deviations above (below) $0 \cdot 05$. Note that both the SMAX and CLOSE tests have the same experimentwise error rate and power. Therefore, Table I reports the simulated error rates for the SMAX (or CLOSE) and CHEN tests only. Since the CHEN test was found to have a relatively conservative level performance, the simulated experimentwise powers for SMAX (or CLOSE) are presented in Tables II and the simulated comparisonwise powers for SMAX and CLOSE are reported in Tables III and IV.

\subsection{Discussion of results}

It can be seen from Table I that the logrank (LR) version of the SMAX or CLOSE test reasonably maintains its level only when the sample size in each group is at least 20. In addition, the SMAX or CLOSE test based on Gehan's (GH) and the Peto-Prentice (PP) statistics hold their levels well across all the situations under consideration. Obviously, the CHEN test tends to be conservative, especially, for the cases with 20 or more observations in each sample.

The power study in Tables II, III and IV indicates that the logrank test is more powerful than either Gehan's or the Peto-Prentice test for exponential distributions. Meanwhile, both the Gehan and Peto-Prentice tests are superior to the logrank test for log-normal distributions. Moreover, although the Peto-Prentice test is better than Gehan's test for exponential distributions, the two tests appear to perform rather similarly for log-normal distributions. 
Table I. Estimated experimentwise error rates for $\alpha=0.05$, uniform censoring distribution $U(0, R)$ and $n_{0}=c, n_{1}=n_{2}=n_{3}=n$

\begin{tabular}{|c|c|c|c|c|c|c|c|}
\hline \multirow[t]{2}{*}{$c, n$} & \multirow[t]{2}{*}{$R$} & \multicolumn{3}{|c|}{ SMAX (or CLOSE) } & \multicolumn{3}{|c|}{ CHEN } \\
\hline & & LR & $\mathrm{GH}$ & $\mathrm{PP}$ & LR & $\mathrm{GH}$ & $\mathrm{PP}$ \\
\hline \multicolumn{8}{|c|}{ Exponential survival distribution } \\
\hline 10,10 & $\begin{array}{l}9 \cdot 901 \\
3 \cdot 185\end{array}$ & $\begin{array}{l}0.065+ \\
0.060+\end{array}$ & $\begin{array}{l}0 \cdot 051 \\
0 \cdot 049\end{array}$ & $\begin{array}{l}0 \cdot 051 \\
0 \cdot 050\end{array}$ & $\begin{array}{l}0.059+ \\
0.053\end{array}$ & $\begin{array}{l}0 \cdot 045 \\
0 \cdot 044\end{array}$ & $\begin{array}{l}0 \cdot 047 \\
0 \cdot 044\end{array}$ \\
\hline 20,20 & $\begin{array}{l}9 \cdot 901 \\
3 \cdot 185\end{array}$ & $\begin{array}{l}0.056 \\
0.052\end{array}$ & $\begin{array}{l}0.053 \\
0 \cdot 046\end{array}$ & $\begin{array}{l}0 \cdot 054 \\
0 \cdot 046\end{array}$ & $\begin{array}{l}0 \cdot 052 \\
0 \cdot 046\end{array}$ & $\begin{array}{l}0.045 \\
0.039-\end{array}$ & $\begin{array}{l}0.046 \\
0.040-\end{array}$ \\
\hline 30,30 & $\begin{array}{l}9 \cdot 901 \\
3 \cdot 185\end{array}$ & $\begin{array}{l}0.055 \\
0.054\end{array}$ & $\begin{array}{l}0.053 \\
0 \cdot 049\end{array}$ & $\begin{array}{l}0.054 \\
0 \cdot 050\end{array}$ & $\begin{array}{l}0.050 \\
0.047\end{array}$ & $\begin{array}{l}0.050 \\
0 \cdot 042-\end{array}$ & $\begin{array}{l}0.049 \\
0.043-\end{array}$ \\
\hline 30,20 & $\begin{array}{l}9 \cdot 901 \\
3 \cdot 185\end{array}$ & $\begin{array}{l}0.051 \\
0.048\end{array}$ & $\begin{array}{l}0 \cdot 045 \\
0 \cdot 044\end{array}$ & $\begin{array}{l}0.046 \\
0.045\end{array}$ & $\begin{array}{l}0.045 \\
0.040-\end{array}$ & $\begin{array}{l}0.035- \\
0.038-\end{array}$ & $\begin{array}{l}0.035- \\
0.038-\end{array}$ \\
\hline \multicolumn{8}{|c|}{ Log-normal survival distribution } \\
\hline 10,10 & $\begin{array}{r}11 \cdot 219 \\
3.756\end{array}$ & $\begin{array}{l}0 \cdot 060+ \\
0 \cdot 062+\end{array}$ & $\begin{array}{l}0.050 \\
0 \cdot 056\end{array}$ & $\begin{array}{l}0 \cdot 051 \\
0 \cdot 056\end{array}$ & $\begin{array}{l}0 \cdot 052 \\
0.056\end{array}$ & $\begin{array}{l}0.044 \\
0.049\end{array}$ & $\begin{array}{l}0.042- \\
0.049\end{array}$ \\
\hline 20,20 & $\begin{array}{r}11.219 \\
3.756\end{array}$ & $\begin{array}{l}0.053 \\
0.056\end{array}$ & $\begin{array}{l}0 \cdot 047 \\
0 \cdot 051\end{array}$ & $\begin{array}{l}0.048 \\
0.052\end{array}$ & $\begin{array}{l}0.045 \\
0 \cdot 055\end{array}$ & $\begin{array}{l}0.041- \\
0.044\end{array}$ & $\begin{array}{l}0.042- \\
0 \cdot 044\end{array}$ \\
\hline 30,30 & $\begin{array}{r}11 \cdot 219 \\
3.756\end{array}$ & $\begin{array}{l}0.049 \\
0.055\end{array}$ & $\begin{array}{l}0.047 \\
0 \cdot 051\end{array}$ & $\begin{array}{l}0.047 \\
0.053\end{array}$ & $\begin{array}{l}0 \cdot 042- \\
0 \cdot 050\end{array}$ & $\begin{array}{l}0.042- \\
0.043-\end{array}$ & $\begin{array}{l}0.042- \\
0.045\end{array}$ \\
\hline 30,20 & $\begin{array}{r}11 \cdot 219 \\
3.756\end{array}$ & $\begin{array}{l}0 \cdot 056 \\
0 \cdot 051\end{array}$ & $\begin{array}{l}0 \cdot 050 \\
0 \cdot 048\end{array}$ & $\begin{array}{l}0.049 \\
0.048\end{array}$ & $\begin{array}{l}0.053 \\
0 \cdot 046\end{array}$ & $\begin{array}{l}0.045 \\
0 \cdot 043\end{array}$ & $\begin{array}{l}0 \cdot 045 \\
0 \cdot 044\end{array}$ \\
\hline
\end{tabular}

$+(-)$ : at least two standard deviations above (below) $\alpha=0.05$

The comparisonwise power study further demonstrates that the CLOSE test is more powerful than the SMAX test when there are two or more treatments better than the control. The apparent superiority of the CLOSE test over the SMAX test occurs when all the treatments are more effective than the control. The SMAX test is slightly better than the CLOSE test when there is only one treatment better than the control and the rest are equally effective as the control. This is because that, in this case, the CLOSE test has a greater likelihood of erroneously claiming that there is more than one treatment better than the control.

\section{DATA ANALYSIS}

In the renal carcinoma (Renca) tumours study mentioned in Section 2, four groups of mice injected with Renca cells were arranged to receive no treatment (control), IL-2 alone (treatment 1), IL-12 alone (treatment 2) and IL-2 plus IL-12 (treatment 3), respectively. Figure 1 presents the Kaplan-Meier ${ }^{18}$ estimates of the survival functions for the four groups of mice.

To assess whether IL-2, IL-12 or IL-2 plus IL-12 displays a greater anti-tumour activity than that exhibited without any agent, the two-sample weighted logrank statistics with the same type of weight function for comparing IL-2, IL-12 or IL-2 plus IL-12 with the control are computed and listed in Table V. However, the plot of the $\log \{-\log$ (Kaplan-Meier survival estimate) $\}$ in Figure 2 suggests that the proportional hazards model may not be appropriate for the pair of 
Table II. Estimated experimentwise powers of SMAX or CLOSE tests for $\alpha=0 \cdot 05$, uniform censoring distribution $U(0, R)$ and $n_{0}=c, n_{1}=n_{2}=n_{3}=20$

\begin{tabular}{|c|c|c|c|c|c|c|c|c|c|c|}
\hline \multirow[t]{2}{*}{$R$} & \multirow[t]{2}{*}{$\theta_{0}$} & \multirow[t]{2}{*}{$\theta_{1}$} & \multirow[t]{2}{*}{$\theta_{2}$} & \multirow[t]{2}{*}{$\theta_{3}$} & \multicolumn{3}{|c|}{$c=20$} & \multicolumn{3}{|c|}{$c=30$} \\
\hline & & & & & LR & GH & PP & LR & GH & PP \\
\hline \multicolumn{11}{|c|}{ Exponential survival distribution } \\
\hline \multirow[t]{5}{*}{$9 \cdot 901$} & 1 & 1 & 1 & $2 \cdot 5$ & 0.676 & 0.570 & 0.585 & 0.749 & 0.626 & 0.635 \\
\hline & 1 & 1 & $1 \cdot 8$ & $2 \cdot 5$ & 0.736 & 0.637 & 0.651 & $0 \cdot 806$ & 0.696 & $0 \cdot 711$ \\
\hline & 1 & 1 & $2 \cdot 5$ & $2 \cdot 5$ & $0 \cdot 841$ & 0.761 & $0 \cdot 774$ & 0.897 & $0 \cdot 818$ & $0 \cdot 828$ \\
\hline & 1 & 1.8 & $2 \cdot 5$ & $2 \cdot 5$ & 0.849 & 0.775 & 0.787 & 0.915 & 0.845 & 0.857 \\
\hline & 1 & $2 \cdot 5$ & $2 \cdot 5$ & $2 \cdot 5$ & 0.909 & $0 \cdot 844$ & $0 \cdot 852$ & 0.949 & 0.893 & $0 \cdot 901$ \\
\hline \multirow[t]{5}{*}{$3 \cdot 185$} & 1 & 1 & 1 & $2 \cdot 5$ & 0.536 & $0 \cdot 448$ & $0 \cdot 482$ & 0.589 & $0 \cdot 481$ & $0 \cdot 519$ \\
\hline & 1 & 1 & $1 \cdot 8$ & $2 \cdot 5$ & 0.593 & 0.508 & 0.542 & 0.655 & 0.568 & 0.607 \\
\hline & 1 & 1 & $2 \cdot 5$ & $2 \cdot 5$ & 0.691 & 0.620 & 0.654 & $0 \cdot 772$ & 0.668 & 0.727 \\
\hline & 1 & $1 \cdot 8$ & $2 \cdot 5$ & $2 \cdot 5$ & 0.715 & 0.640 & $0 \cdot 671$ & $0 \cdot 790$ & 0.715 & $0 \cdot 752$ \\
\hline & 1 & $2 \cdot 5$ & $2 \cdot 5$ & $2 \cdot 5$ & 0.773 & $0 \cdot 704$ & 0.735 & 0.847 & 0.782 & $0 \cdot 815$ \\
\hline \multicolumn{11}{|c|}{ Log-normal survival distribution } \\
\hline \multirow[t]{5}{*}{11.219} & 0 & 0 & 0 & 0.5 & 0.748 & 0.787 & 0.787 & 0.822 & 0.859 & 0.861 \\
\hline & 0 & 0 & $0 \cdot 3$ & 0.5 & 0.771 & $0 \cdot 815$ & $0 \cdot 818$ & $0 \cdot 841$ & 0.885 & 0.888 \\
\hline & 0 & 0 & 0.5 & 0.5 & 0.873 & 0.910 & 0.910 & 0.918 & 0.949 & 0.949 \\
\hline & 0 & $0 \cdot 3$ & $0 \cdot 5$ & 0.5 & 0.877 & 0.921 & 0.921 & 0.931 & 0.954 & $0 \cdot 953$ \\
\hline & 0 & 0.5 & 0.5 & 0.5 & $0 \cdot 911$ & 0.947 & 0.947 & 0.958 & 0.974 & 0.973 \\
\hline \multirow[t]{5}{*}{3.756} & 0 & 0 & 0 & 0.5 & 0.662 & 0.688 & 0.688 & 0.724 & 0.758 & 0.763 \\
\hline & 0 & 0 & $0 \cdot 3$ & 0.5 & 0.711 & 0.741 & 0.741 & 0.765 & 0.801 & 0.802 \\
\hline & 0 & 0 & 0.5 & 0.5 & $0 \cdot 806$ & 0.842 & 0.844 & $0 \cdot 867$ & 0.900 & $0 \cdot 900$ \\
\hline & 0 & 0.3 & 0.5 & 0.5 & $0 \cdot 814$ & 0.844 & 0.845 & 0.879 & 0.913 & 0.915 \\
\hline & 0 & $0 \cdot 5$ & 0.5 & 0.5 & 0.865 & $0 \cdot 900$ & 0.904 & 0.924 & 0.942 & $0 \cdot 944$ \\
\hline
\end{tabular}

IL-12 and the control, since the vertical distance between the two plots is not a constant. The survival plot in Figure 1 further suggests that the hazards of IL-12 and the control differ at early times. Therefore, we employ the two-sample Peto-Prentice statistic for comparing IL-12 and the control, but utilize the unweighted logrank statistics for comparing IL-12 and the control as well as IL-2 plus IL-12 and the control. The relevant statistics are also reported in Table V. In addition, to evaluate whether the combined administration of IL-2 and IL-12 yields greater anti-tumour activity against the established Renca tumours than either IL-12 or IL-2 alone, two-sample weighted logrank statistics for comparing IL-2 plus IL-12 (control) with IL-2 along (treatment 1) and IL-12 alone (treatment 2), respectively, are calculated and reported in Table V.

An empirical observation from Table $\mathrm{V}$ is that the estimated correlations are all relatively close to 0.5 , which accounts for why we compare the $U_{i}$ 's with the appropriate level $\alpha=0.05$ critical values $(z \max (3,0.05) \approx 2.06, z \max (2,0.05) \approx 1.92$ and $z \max (1,0.05)=1.65)$ found in Gupta. ${ }^{17}$ The critical value used in the CHEN test is $z(0 \cdot 017)=2 \cdot 12$ for three treatments versus a control, while it is $z(0.025)=1.96$ for comparing two treatments with a control. Both the SMAX and CHEN tests claim that, under $\alpha=0 \cdot 05$, when compared with the no treatment control, only IL-2 plus IL-12 has an effect in regression of Renca tumour, since 3.724, 3.552 and 3.508 are greater than $2 \cdot 12$, but $1 \cdot 788,2 \cdot 004$ and $2 \cdot 024$ are all less than 2.06. The CLOSE test based on logrank statistics $(1.788<1.92)$ reaches the same conclusion as that of the SMAX and CHEN tests, while the one based on the Gehan or Peto-Prentice statistics $(2.004>1.92$ and 2.024 $>1.92$, but 
Table III. Estimated comparisonwise powers for $\alpha=0.05$, exponential survival distribution, uniform censoring distribution $U(0, R)$ and $n_{0}=c, n_{1}=n_{2}=n_{3}=20$

\begin{tabular}{|c|c|c|c|c|c|c|c|c|c|c|}
\hline \multirow[t]{2}{*}{$R$} & \multirow[t]{2}{*}{$\theta_{0}$} & \multirow[t]{2}{*}{$\theta_{1}$} & \multirow[t]{2}{*}{$\theta_{2}$} & \multirow[t]{2}{*}{$\theta_{3}$} & \multicolumn{3}{|c|}{ SMAX } & \multicolumn{3}{|c|}{ CLOSE } \\
\hline & & & & & LR & $\mathrm{GH}$ & PP & LR & GH & PP \\
\hline $\begin{array}{l}c=20 \\
9 \cdot 901\end{array}$ & $\begin{array}{l}1 \\
1 \\
1 \\
1 \\
1\end{array}$ & $\begin{array}{l}1 \\
1 \\
1 \\
1 \cdot 8 \\
2 \cdot 5\end{array}$ & $\begin{array}{l}1 \\
1 \cdot 8 \\
2 \cdot 5 \\
2 \cdot 5 \\
2 \cdot 5\end{array}$ & $\begin{array}{l}2 \cdot 5 \\
2 \cdot 5 \\
2 \cdot 5 \\
2 \cdot 5 \\
2 \cdot 5\end{array}$ & $\begin{array}{l}0 \cdot 641 \\
0 \cdot 273 \\
0 \cdot 485 \\
0 \cdot 269 \\
0 \cdot 412\end{array}$ & $\begin{array}{l}0.534 \\
0 \cdot 198 \\
0.364 \\
0 \cdot 171 \\
0 \cdot 268\end{array}$ & $\begin{array}{l}0 \cdot 547 \\
0 \cdot 202 \\
0 \cdot 375 \\
0 \cdot 177 \\
0 \cdot 280\end{array}$ & $\begin{array}{l}0 \cdot 630 \\
0 \cdot 307 \\
0.521 \\
0 \cdot 425 \\
0.599\end{array}$ & $\begin{array}{l}0 \cdot 523 \\
0 \cdot 226 \\
0 \cdot 399 \\
0 \cdot 310 \\
0 \cdot 447\end{array}$ & $\begin{array}{l}0 \cdot 535 \\
0 \cdot 231 \\
0 \cdot 412 \\
0 \cdot 316 \\
0 \cdot 460\end{array}$ \\
\hline $3 \cdot 185$ & $\begin{array}{l}1 \\
1 \\
1 \\
1 \\
1\end{array}$ & $\begin{array}{l}1 \\
1 \\
1 \\
1 \cdot 8 \\
2 \cdot 5\end{array}$ & $\begin{array}{l}1 \\
1 \cdot 8 \\
2 \cdot 5 \\
2 \cdot 5 \\
2 \cdot 5\end{array}$ & $\begin{array}{l}2 \cdot 5 \\
2 \cdot 5 \\
2 \cdot 5 \\
2 \cdot 5 \\
2 \cdot 5\end{array}$ & $\begin{array}{l}0 \cdot 497 \\
0 \cdot 189 \\
0 \cdot 335 \\
0 \cdot 162 \\
0 \cdot 251\end{array}$ & $\begin{array}{l}0 \cdot 408 \\
0 \cdot 135 \\
0 \cdot 240 \\
0 \cdot 099 \\
0 \cdot 166\end{array}$ & $\begin{array}{l}0 \cdot 443 \\
0 \cdot 152 \\
0 \cdot 270 \\
0 \cdot 114 \\
0 \cdot 195\end{array}$ & $\begin{array}{l}0 \cdot 486 \\
0 \cdot 212 \\
0 \cdot 363 \\
0 \cdot 282 \\
0 \cdot 418\end{array}$ & $\begin{array}{l}0 \cdot 396 \\
0 \cdot 151 \\
0 \cdot 266 \\
0 \cdot 200 \\
0 \cdot 314\end{array}$ & $\begin{array}{l}0 \cdot 431 \\
0 \cdot 170 \\
0 \cdot 298 \\
0 \cdot 221 \\
0 \cdot 343\end{array}$ \\
\hline $\begin{array}{l}c=30 \\
9 \cdot 901\end{array}$ & $\begin{array}{l}1 \\
1 \\
1 \\
1 \\
1\end{array}$ & $\begin{array}{l}1 \\
1 \\
1 \\
1 \cdot 8 \\
2 \cdot 5\end{array}$ & $\begin{array}{l}1 \\
1 \cdot 8 \\
2 \cdot 5 \\
2 \cdot 5 \\
2 \cdot 5\end{array}$ & $\begin{array}{l}2 \cdot 5 \\
2 \cdot 5 \\
2 \cdot 5 \\
2 \cdot 5 \\
2 \cdot 5\end{array}$ & $\begin{array}{l}0 \cdot 716 \\
0 \cdot 327 \\
0 \cdot 594 \\
0 \cdot 305 \\
0 \cdot 510\end{array}$ & $\begin{array}{l}0.609 \\
0.227 \\
0.433 \\
0.189 \\
0.329\end{array}$ & $\begin{array}{l}0 \cdot 620 \\
0 \cdot 234 \\
0 \cdot 448 \\
0 \cdot 198 \\
0 \cdot 341\end{array}$ & $\begin{array}{l}0 \cdot 702 \\
0 \cdot 364 \\
0 \cdot 621 \\
0 \cdot 470 \\
0 \cdot 689\end{array}$ & $\begin{array}{l}0 \cdot 596 \\
0 \cdot 263 \\
0 \cdot 474 \\
0 \cdot 339 \\
0 \cdot 519\end{array}$ & $\begin{array}{l}0 \cdot 606 \\
0 \cdot 271 \\
0 \cdot 488 \\
0 \cdot 350 \\
0 \cdot 533\end{array}$ \\
\hline $3 \cdot 185$ & $\begin{array}{l}1 \\
1 \\
1 \\
1 \\
1\end{array}$ & $\begin{array}{l}1 \\
1 \\
1 \\
1 \cdot 8 \\
2 \cdot 5\end{array}$ & $\begin{array}{l}1 \\
1 \cdot 8 \\
2 \cdot 5 \\
2 \cdot 5 \\
2 \cdot 5\end{array}$ & $\begin{array}{l}2.5 \\
2.5 \\
2.5 \\
2.5 \\
2.5\end{array}$ & $\begin{array}{l}0 \cdot 545 \\
0 \cdot 216 \\
0 \cdot 389 \\
0 \cdot 180 \\
0 \cdot 294\end{array}$ & $\begin{array}{l}0 \cdot 452 \\
0 \cdot 152 \\
0 \cdot 271 \\
0 \cdot 108 \\
0 \cdot 181\end{array}$ & $\begin{array}{l}0 \cdot 485 \\
0 \cdot 174 \\
0 \cdot 310 \\
0 \cdot 128 \\
0 \cdot 217\end{array}$ & $\begin{array}{l}0.533 \\
0 \cdot 250 \\
0 \cdot 424 \\
0.327 \\
0.479\end{array}$ & $\begin{array}{l}0 \cdot 441 \\
0 \cdot 184 \\
0 \cdot 309 \\
0 \cdot 225 \\
0 \cdot 346\end{array}$ & $\begin{array}{l}0 \cdot 473 \\
0 \cdot 204 \\
0 \cdot 348 \\
0 \cdot 255 \\
0 \cdot 392\end{array}$ \\
\hline
\end{tabular}

$1.564<1.65$ ad $1.585<1.65$ ) claims that both IL-12 alone and IL-2 in combination with IL-12 exhibit anti-tumour activity against the Renca tumours. However, it is not the case that all the hazard differences occur at early times. Therefore, according to the procedure based on different types of two-sample weighted logrank statistics $(3.724>2.06,2.024>1.92$ and $1.696>1.65)$, we conclude that IL-2, IL-12 and IL-2 plus IL-12 all display a greater anti-tumour activity than that exhibited without any agent. Note that, for such a conclusion reached by the SMAX test based on different types of weighted logrank statistics $\left(d_{1}=1, d_{2}=2, d_{3}=3\right)$, the approximate $p$-value is $p($ SMAX; $1,2,3)=1-P\left\{Z_{1} \leqslant 1 \cdot 696, Z_{2} \leqslant 1 \cdot 696, Z_{3} \leqslant 1 \cdot 696\right\}=0 \cdot 108$. However, we observe $p^{\prime}(1)=1-P\left\{Z_{1} \leqslant 1.696\right\}=0.045, p^{\prime}(2)=1-P\left\{Z_{1} \leqslant 2.024, Z_{2} \leqslant 2.024\right\}=0.039$ and $p^{\prime}(3)=$ $1-P\left\{Z_{1} \leqslant 3 \cdot 724, Z_{2} \leqslant 3 \cdot 724, Z_{3} \leqslant 3 \cdot 724\right\}=0 \cdot 0002$. The approximate $p$-value of the closed test in (6) with the conclusion that all the three treatments are more effective than the control is then obtained as $p($ CLOSE; $1,2,3)=\max \left\{p^{\prime}\left(d_{1}\right), p^{\prime}\left(d_{2}\right), p^{\prime}\left(d_{3}\right)\right\}=0 \cdot 045$.

To assess the appropriateness of the scale-change models for the three treatments versus a control setting, Figure 3 displays the quantile-quantile plots (Wei and Gail ${ }^{10}$ ) for IL-2 versus the control, IL-12 versus the control, and IL-2 plus IL-12 versus the control. Note that quantile-quantile pairs do not deviate from linearity. Let $T_{0}, T_{1}, T_{3}$ represent the survival times for the control, IL-2, IL-12 and IL-2 plus IL-12 groups, respectively. The least squared fits 
Table IV. Estimated comparisonwise powers for $\alpha=0 \cdot 05$, log-normal survival distribution, uniform censoring distribution $U(0, R)$ and $n_{0}=c, n_{1}=n_{2}=n_{3}=20$

\begin{tabular}{|c|c|c|c|c|c|c|c|c|c|c|}
\hline \multirow[t]{2}{*}{$R$} & \multirow[t]{2}{*}{$\theta_{0}$} & \multirow[t]{2}{*}{$\theta_{1}$} & \multirow[t]{2}{*}{$\theta_{2}$} & \multirow[t]{2}{*}{$\theta_{3}$} & \multicolumn{3}{|c|}{ SMAX } & \multicolumn{3}{|c|}{ CLOSE } \\
\hline & & & & & LR & $\mathrm{GH}$ & PP & LR & GH & PP \\
\hline $\begin{array}{l}c=20 \\
11 \cdot 219\end{array}$ & $\begin{array}{l}0 \\
0 \\
0 \\
0 \\
0\end{array}$ & $\begin{array}{l}0 \\
0 \\
0 \\
0 \cdot 3 \\
0 \cdot 5\end{array}$ & $\begin{array}{l}0 \\
0 \cdot 3 \\
0 \cdot 5 \\
0 \cdot 5 \\
0.5\end{array}$ & $\begin{array}{l}0 \cdot 5 \\
0 \cdot 5 \\
0 \cdot 5 \\
0 \cdot 5 \\
0.5\end{array}$ & $\begin{array}{l}0 \cdot 702 \\
0 \cdot 306 \\
0 \cdot 614 \\
0 \cdot 305 \\
0 \cdot 563\end{array}$ & $\begin{array}{l}0 \cdot 751 \\
0.323 \\
0.653 \\
0.313 \\
0.598\end{array}$ & $\begin{array}{l}0 \cdot 754 \\
0 \cdot 324 \\
0 \cdot 655 \\
0 \cdot 315 \\
0 \cdot 600\end{array}$ & $\begin{array}{l}0.698 \\
0.325 \\
0.640 \\
0.457 \\
0.718\end{array}$ & $\begin{array}{l}0 \cdot 739 \\
0 \cdot 360 \\
0 \cdot 683 \\
0 \cdot 483 \\
0 \cdot 763\end{array}$ & $\begin{array}{l}0.742 \\
0.361 \\
0.684 \\
0.485 \\
0.763\end{array}$ \\
\hline 3.576 & $\begin{array}{l}0 \\
0 \\
0 \\
0 \\
0\end{array}$ & $\begin{array}{l}0 \\
0 \\
0 \\
0 \cdot 3 \\
0 \cdot 5\end{array}$ & $\begin{array}{l}0 \\
0 \cdot 3 \\
0 \cdot 5 \\
0 \cdot 5 \\
0.5\end{array}$ & $\begin{array}{l}0.5 \\
0.5 \\
0.5 \\
0.5 \\
0.5\end{array}$ & $\begin{array}{l}0.639 \\
0.258 \\
0.515 \\
0.257 \\
0.444\end{array}$ & $\begin{array}{l}0.670 \\
0.263 \\
0.533 \\
0.256 \\
0.454\end{array}$ & $\begin{array}{l}0.671 \\
0.267 \\
0.537 \\
0.257 \\
0.459\end{array}$ & $\begin{array}{l}0.626 \\
0.281 \\
0.538 \\
0.393 \\
0.605\end{array}$ & $\begin{array}{l}0.655 \\
0 \cdot 298 \\
0.566 \\
0 \cdot 422 \\
0.635\end{array}$ & $\begin{array}{l}0.657 \\
0.300 \\
0.568 \\
0.427 \\
0.635\end{array}$ \\
\hline $\begin{array}{l}c=30 \\
11 \cdot 219\end{array}$ & $\begin{array}{l}0 \\
0 \\
0 \\
0 \\
0\end{array}$ & $\begin{array}{l}0 \\
0 \\
0 \\
0 \cdot 3 \\
0 \cdot 5\end{array}$ & $\begin{array}{l}0 \\
0 \cdot 3 \\
0 \cdot 5 \\
0 \cdot 5 \\
0.5\end{array}$ & $\begin{array}{l}0 \cdot 5 \\
0 \cdot 5 \\
0 \cdot 5 \\
0 \cdot 5 \\
0 \cdot 5\end{array}$ & $\begin{array}{l}0.778 \\
0.343 \\
0.677 \\
0.332 \\
0.624\end{array}$ & $\begin{array}{l}0.817 \\
0.370 \\
0.730 \\
0.358 \\
0.676\end{array}$ & $\begin{array}{l}0 \cdot 817 \\
0 \cdot 371 \\
0 \cdot 731 \\
0 \cdot 359 \\
0.676\end{array}$ & $\begin{array}{l}0 \cdot 767 \\
0 \cdot 375 \\
0 \cdot 700 \\
0.494 \\
0.778\end{array}$ & $\begin{array}{l}0 \cdot 801 \\
0 \cdot 413 \\
0 \cdot 761 \\
0 \cdot 545 \\
0 \cdot 825\end{array}$ & $\begin{array}{l}0.801 \\
0.413 \\
0.761 \\
0.546 \\
0.825\end{array}$ \\
\hline 3.576 & $\begin{array}{l}0 \\
0 \\
0 \\
0 \\
0\end{array}$ & $\begin{array}{l}0 \\
0 \\
0 \\
0 \cdot 3 \\
0 \cdot 5\end{array}$ & $\begin{array}{l}0 \\
0.3 \\
0.5 \\
0.5 \\
0.5\end{array}$ & $\begin{array}{l}0.5 \\
0.5 \\
0.5 \\
0.5 \\
0.5\end{array}$ & $\begin{array}{l}0 \cdot 700 \\
0 \cdot 280 \\
0 \cdot 560 \\
0 \cdot 261 \\
0 \cdot 493\end{array}$ & $\begin{array}{l}0.736 \\
0.291 \\
0.595 \\
0 \cdot 276 \\
0.520\end{array}$ & $\begin{array}{l}0.737 \\
0.295 \\
0.602 \\
0.279 \\
0.530\end{array}$ & $\begin{array}{l}0.686 \\
0.311 \\
0.595 \\
0.425 \\
0.677\end{array}$ & $\begin{array}{l}0 \cdot 723 \\
0 \cdot 332 \\
0 \cdot 640 \\
0 \cdot 450 \\
0 \cdot 716\end{array}$ & $\begin{array}{l}0.726 \\
0.335 \\
0.641 \\
0.452 \\
0.718\end{array}$ \\
\hline
\end{tabular}

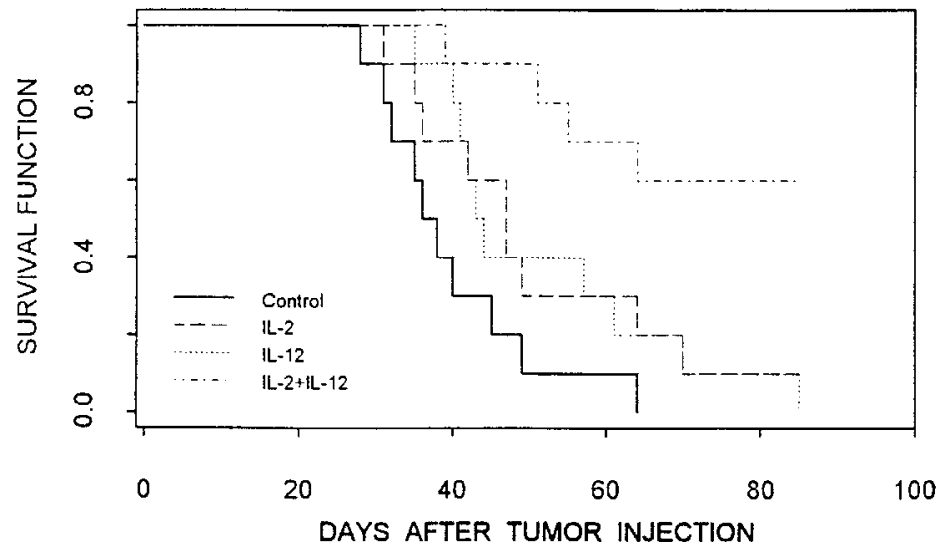

Figure 1. Kaplan-Meier estimates for the interleukin-tumour example 
Table V. Summary statistics for the interleukin-tumour example

\begin{tabular}{|c|c|c|c|c|c|c|}
\hline \multirow[t]{2}{*}{ Statistics } & \multicolumn{2}{|r|}{ Logrank } & \multicolumn{2}{|r|}{ Gehan } & \multicolumn{2}{|c|}{ Peto-Prentice } \\
\hline & \multicolumn{6}{|c|}{1 for $I L-2,2$ for $I L-12$ and 3 for $I L-2+I L-12$} \\
\hline $\mathrm{U}_{1}\left(U_{01}, s_{11}\right)$ & 1.696 & $(3 \cdot 270,3 \cdot 715)$ & $1 \cdot 564$ & $(2 \cdot 050,1 \cdot 719)$ & $1 \cdot 585$ & $(1 \cdot 854,1 \cdot 368)$ \\
\hline $\mathrm{U}_{2}\left(U_{02}, s_{22}\right)$ & $1 \cdot 788$ & $(3 \cdot 560,3 \cdot 962)$ & $2 \cdot 004$ & $(2.600,1.683)$ & $2 \cdot 024$ & $(2 \cdot 394,1.399)$ \\
\hline $\mathrm{U}_{3}\left(U_{03}, s_{33}\right)$ & $3 \cdot 724$ & $(5 \cdot 975,2 \cdot 575)$ & $3 \cdot 552$ & $(4 \cdot 350,1 \cdot 500)$ & $3 \cdot 508$ & $(4 \cdot 014,1 \cdot 309)$ \\
\hline \multirow{3}{*}{$\mathbf{S}$} & $1 \cdot 000$ & $0.553 \quad 0.527$ & $1 \cdot 000$ & $0.476 \quad 0.459$ & $1 \cdot 000$ & $0 \cdot 479 \quad 0 \cdot 460$ \\
\hline & & $1.000 \quad 0.524$ & & $1.000 \quad 0.453$ & & $1.000 \quad 0.458$ \\
\hline & & $1 \cdot 000$ & & $1 \cdot 000$ & & $1 \cdot 000$ \\
\hline $\mathrm{U}_{1}$ : Logrank & 1.696 & \multirow{3}{*}{\multicolumn{2}{|c|}{$\mathbf{S}=$}} & $1.000 \quad 0.478$ & $0 \cdot 527$ & \\
\hline $\mathrm{U}_{2}$ : Peto-Prentice & $2 \cdot 024$ & & & $1 \cdot 000$ & $0 \cdot 456$ & \\
\hline $\mathrm{U}_{3}:$ Logrank & 3.724 & & & & $1 \cdot 000$ & \\
\hline \multicolumn{7}{|c|}{ (b) 0 for $I L-2+I L-12,1$ for $I L-2$ and 2 for $I L-12$} \\
\hline $\mathrm{U}_{1}\left(U_{10}, s_{11}\right)$ & $2 \cdot 523$ & $(4 \cdot 252,2 \cdot 840)$ & $2 \cdot 491$ & $(3 \cdot 150,1 \cdot 599)$ & $2 \cdot 456$ & $(2 \cdot 867,1 \cdot 353)$ \\
\hline \multirow[t]{2}{*}{$\mathrm{U}_{2}\left(U_{20}, s_{22}\right)$} & $2 \cdot 819$ & $(4 \cdot 941,3 \cdot 074)$ & $2 \cdot 424$ & $(3 \cdot 100,1 \cdot 635)$ & $2 \cdot 389$ & $(2 \cdot 853,1 \cdot 427)$ \\
\hline & $1 \cdot 000$ & $0 \cdot 451$ & $1 \cdot 000$ & $0 \cdot 493$ & $1 \cdot 000$ & $0 \cdot 519$ \\
\hline $\mathbf{S}$ & $1 \cdot 000$ & $1 \cdot 000$ & & $1 \cdot 000$ & & \\
\hline
\end{tabular}

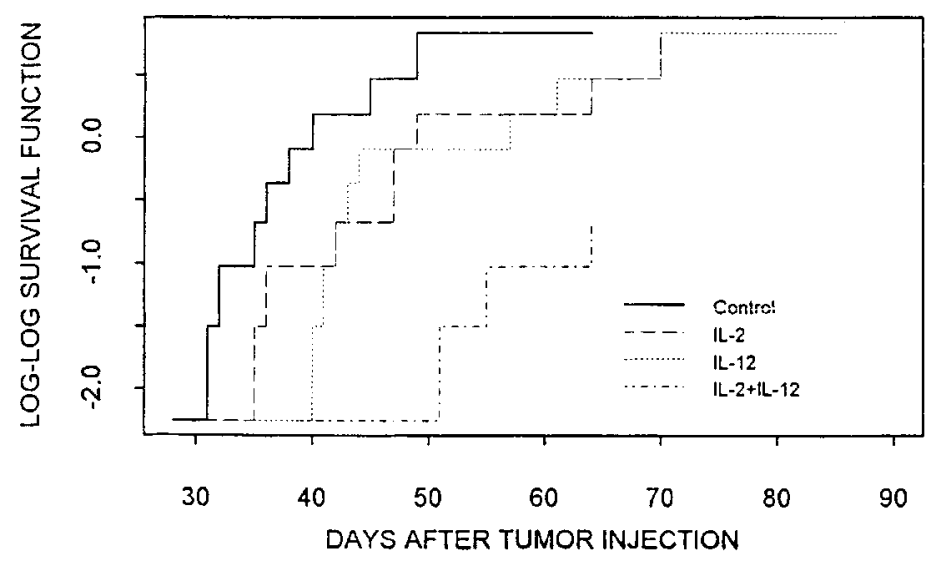

Figure 2. The $\log \{-\log ($ Kaplan-Meier estimate $)\}$ for the interleukin-tumour example

suggested scale-change models for $\left(T_{0}-27, T_{1}-27\right),\left(T_{0}, T_{2}\right)$ and $\left.T_{0}-24, T_{3}-24\right)$. Let $\gamma_{1}, \gamma_{2}$ and $\gamma_{3}$ be the ratios of the scale parameters for $T_{1}-27$ and $T_{0}-27, T_{2}$ and $T_{0}$, and $T_{3}-25$ and $T_{0}-25$, respectively. A 95 per cent confidence set for $\left(\gamma_{1}, \gamma_{2}, \gamma_{3}\right),\left\{\left(\gamma_{1}, \gamma_{2}, \gamma_{3}\right): \gamma_{1} \geqslant \gamma_{1}, \gamma_{2} \geqslant \gamma_{2}, \gamma_{3} \geqslant \gamma_{3}\right\}$ based on the procedure with difference types of two-sample weighted logrank statistics then yields $\left(\gamma_{1}, \gamma_{2}, \gamma_{3}\right)=(0.91,0.99,2.51)$. Note that these findings are in a good agreement with the results of the SMAX test $(3 \cdot 724>2 \cdot 06$, but $2 \cdot 024<2 \cdot 06)$.

Note that the log-log survival plot in Figure 2 reveals that the proportional hazards model is reasonable for the pair of IL-2 alone and IL-2 plus IL-12 or IL-12 alone and IL-2 plus IL-12. Hence, based on two-sample unweighted logrank statistics, the SMAX and CLOSE tests claim, at 


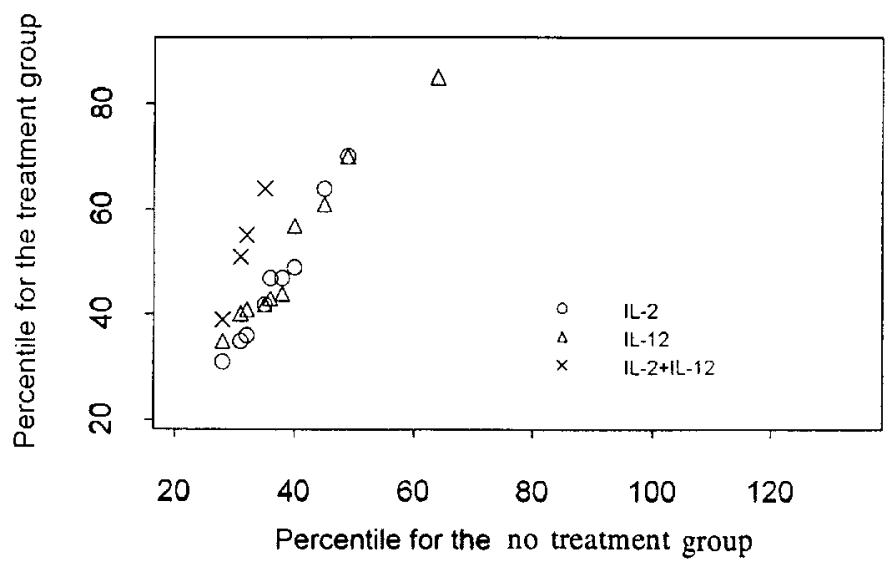

Figure 3. Quantile-quantile plot for the interleukin-tumour example

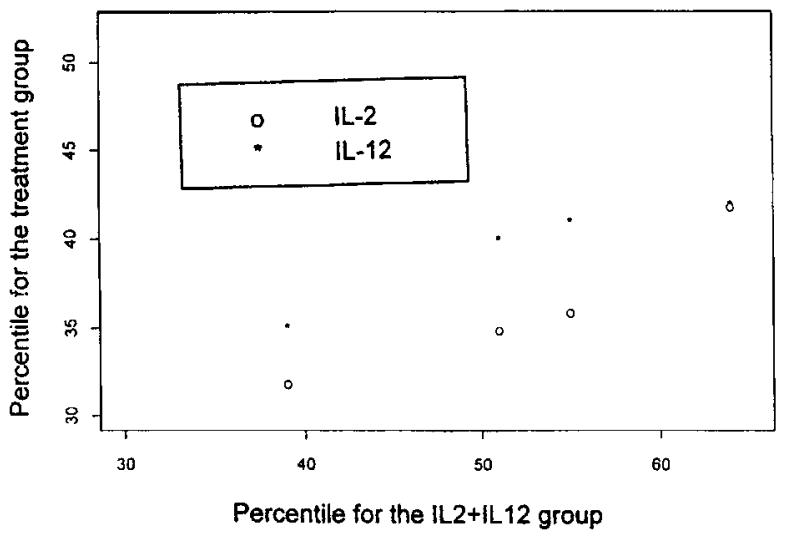

Figure 4. Quantile-quantile plot for the interleukin-tumour example

$\alpha=0 \cdot 05$, that IL-2 plus IL-12 yields better anti-tumour activity against the Renca tumours than IL-2 or IL-12 alone $(2.819>1.92$ and $2.525>1.92)$. Moreover, for such a conclusion, the approximate $p$-value of the SMAX test is $p(\operatorname{SMAX} ; 1,2)=1-P\left\{Z_{1} \leqslant 2 \cdot 525\right.$, $\left.Z_{2} \leqslant 2.525\right\}=0.011$, while the values of $p^{\prime}(1)=1-P\left\{Z_{1} \leqslant 2.525\right\}=0.006$ and $p^{\prime}(2)=$ $1-P\left\{Z_{1} \leqslant 2 \cdot 819, Z_{2} \leqslant 2 \cdot 819\right\}=0.005$ produce an approximate $p$-value of the closed test in (6) as $p($ CLOSE; 1,2$)=\max \left\{p^{\prime}\left(d_{1}\right), p^{\prime}\left(d_{2}\right)\right\}=0.006$.

The quantile-quantile plots for IL-2 versus IL-2 plus IL-12 versus IL-2 plus IL-12 given in Figure 4 also support the scale-change model. Let $T_{0}, T_{1}$ and $T_{2}$ represent the survival times for the IL-2 plus IL-12, IL-2 and IL-12 groups, respectively. The least squared fits suggested scale-change models for $\left(T_{0}-26, T_{1}-26\right),\left(T_{0}-34, T_{2}-34\right)$. Let $\gamma_{1}$ and $\gamma_{2}$ be the ratios of the scale parameters for $T_{0}-26$ and $T_{1}-26$, and $T_{0}-34$ and $T_{2}-34$, respectively. A95 per cent confidence set for $\left(\gamma_{1}, \gamma_{2}\right),\left\{\left(\gamma_{1}, \gamma_{2}: \gamma_{1} \geqslant \gamma_{1}, \gamma_{2} \geqslant \gamma_{2}\right\}\right.$ based on the SMAX test with unweighted logrank statistics then 
yields $\left(\gamma_{1}, \gamma_{2}\right)=(1 \cdot 34,1 \cdot 88)$. These findings confirm, again, the results based on the unweighted logrank SMAX test for comparing IL-2 and IL-12 with IL-2 plus IL-12.

\section{CONCLUSION}

This paper presents a generalized Steel's test (SMAX) and a closed multiple testing procedure (CLOSE), which is actually a modification of the generalized Steel's test, based on two-sample weighted logrank statistics to determine which treatments are more effective than (or different from) the control when survival data are subject to random right-censorship. When the scalechange model is appropriate for such comparisons, a confidence set on the basis of the SMAX test for the scale-changes of each treatment group and the control is suggested. However, if only a significance testing procedure is required, the CLOSE test is recommended, since it has a better comparisonwise power performance than the SMAX test in most practical situations. The $p$-values of the testing procedures are also provided which measure the strength of the evidence for the conclusion that a certain treatment is more effective that (or different from) the control.

The appropriate choice of the two-sample weighted logrank statistics in the treatments versus a control setting is the same as that in the two-sample problem which has been extensively discussed (see, for example, Fleming and Harrington ${ }^{9}$ ). The unweighted logrank statistic should be used when the assumption of proportional hazards is tenable. For testing against early hazard differences, both the Gehan and Peto-Prentice statistics are better than the logrank statistic. However, according to Anderson et al., ${ }^{22}$ the weight function used in the Peto-Prentice test depends only on the survival experience, meanwhile the Gehan test uses a weight function that depends on survivals as well as censorings. Therefore, we suggest employing the two-sample Peto-Prentice statistic in comparing the corresponding treatment with the control when their hazards are different at early times. For the situation where hazard difference occurs at late times, according to the suggestions made by Fleming and Harrington, ${ }^{9}$ the appropriate weight function $W_{i}\left(T_{k}\right)$ used in (1) and (2) should be $\left\{1-\right.$ the Kaplan-Meier ${ }^{18}$ survival estimate $\}$. Finally, the $\log -\log$ survival plot shown in Figure 2 is usually employed for checking the proportional hazards model. The survival plots presented in Figure 1 should be helpful for assessing if the hazard difference occurs at early or late times.

\section{APPENDIX}

\section{Asymptotic null distribution of $\left(U_{1}, U_{2}, \ldots, U_{m}\right)$}

Let $T_{1}<\cdots<T_{L}$ denote the ordered observed distinct death times in the sample formed by combining the $i$ th, $j$ th and control groups, Let $d_{u k}$ and $Y_{u k}, k=1, \ldots, L, u=0, i, j$, denote the number of observed deaths and number at risk, respectively, in sample $u$ at time $T_{k}$. Set $Y_{+1 k}=Y_{0 k}+Y_{1 k}, 1=i, j, Y_{++k}=Y_{0 k}+Y_{i k}+Y_{j k}$, and $d_{++k}=d_{0 k}+d_{i k}+d_{j k}$. For the three weight functions considered herein and under some regular conditions. Chen ${ }^{9}$ showed that the null $\left(H_{0}\right)$ asymptotic distribution of $\left(U_{1}, U_{2}, \ldots, U_{m}\right)$ is an $m$-variate normal with mean zero and covariance matrix $\Sigma=\left(\rho_{i j}\right)$, which can be consistently estimated by $\mathbf{S}=\left(s_{i j} / \sqrt{ } s_{i i} s_{i j}\right)$, where $s_{i i}$ are stated in (2) and

$$
s_{i j}=\sum_{k=1}^{L} W_{i}\left(T_{k}\right) W_{j}\left(T_{k}\right) v_{k}, \quad i \neq j=1,2, \ldots, m
$$

with $v_{k}=d_{++k} Y_{0 k} Y_{i k} Y_{j k}\left(Y_{++k}-d_{++k}\right) /\left\{Y_{+i k} Y_{+j k} Y_{++k}\left(Y_{++k}-1\right)\right\}, k=1,2, \ldots, L$. 


\section{Description of left-sided and two-sided comparisons}

When the control is expected to be at least as good as the control, the two-sample weighted logrank statistics employed in the multiple testing procedures in (3), (4) and (6) would be the ones which compare the control with the $i$ th treatment, namely

$$
U_{i 0}=\sum_{k=1}^{L} W_{i}\left(T_{k}\right)\left(d_{i k}-e_{i k}\right), \quad i=1,2, \ldots, m
$$

where $e_{i k}=d_{+k} Y_{i k} / Y_{+k}$. Let $\gamma_{i}=\theta_{0} / \theta_{i}, i=1,2, \ldots, m$. Then, an approximate $(1-\alpha) \times 100$ per cent confidence set for the $\gamma=\left(\gamma_{1}, \gamma_{2}, \ldots, \gamma_{m}\right)$ can be obtained as specified in (5), but replacing $h\left(\mathbf{X}_{0}, \mathbf{X}_{i} / a, \Delta_{0}, \Delta_{i}\right)$ and $V\left(\mathbf{X}_{0}, \mathbf{X}_{i} / a, \Delta_{0}, \Delta_{i}\right)$ with $h\left(\mathbf{X}_{i}, \mathbf{X}_{0} / a, \Delta_{i}, \Delta_{0}\right)$ and $V\left(\mathbf{X}_{i}, \mathbf{X}_{0} / a, \Delta_{i}, \Delta_{0}\right)$, respectively. Under the two-sided alternative that there is at least one treatment which is different from the control, the appropriate statistics in the generalized Steel's test are $\left|U_{i}\right|, i=1,2, \ldots, m$, where $|x|$ is the absolute value of $x$. The corresponding critical value, denoted by $|z| \max (m, \alpha)$, is the upper $\alpha$ th percentile of the distribution of $\max \left(\left|Z_{1}\right|,\left|Z_{2}\right|, \ldots,\left|Z_{m}\right|\right)$, where $\left(Z_{1}, Z_{2}, \ldots, Z_{m}\right)$ is, again, an $m$-variate normal vector with mean zero and covariance matrix $\mathbf{S}$. For a common censoring distribution and equal sample sizes, some values of the $|z| \max (m, \alpha)$ can be found in Dunnett. ${ }^{23}$ Let $|U|_{(1)}<|U|_{(2)}<\cdots<\cdots|U|_{(m)}$ be the order statistics of the $\left|U_{i}\right|$ 's. The closed testing procedure for the two-sided alternative is then the same as in (6) except that the $U_{(i)}$ and $z \max ((i), \alpha)$ are replaced by $|U|_{(i)}$ and $|z| \max ((i) \alpha)$, respectively. Moreover, an approximate $(1-\alpha) \times 100$ per cent test-based confidence set for the ratios of the scale parameters $\gamma=\left(\gamma_{1}\right.$, $\left.\gamma_{2}, \ldots, \gamma_{m}\right)$, where $\gamma_{i}=\theta_{i} / \theta_{0}, i=1,2, \ldots, m$, in a scale-change model is given by

$$
C(\gamma)=\left\{\left(\gamma_{1}, \gamma_{2}, \ldots, \gamma_{m}\right): \gamma_{i} \leqslant \gamma_{i} \leqslant \bar{\gamma}_{i}, \quad i=1,2, \ldots, m\right\}
$$

where $\gamma_{i}$ is the smallest value of a such that

$$
h\left(\mathbf{X}_{0}, \mathbf{X}_{i} / a, \Delta_{0}, \Delta_{i}\right) \leqslant|z| \max (m, \alpha) \sqrt{ }\left\{V\left(\mathbf{X}_{0}, \mathbf{X}_{i} / a, \Delta_{0}, \Delta_{i}\right)\right\}
$$

and $\bar{\gamma}_{i}$ is the largest value of $b$ satisfying

$$
h\left(\mathbf{X}_{0}, \mathbf{X}_{i} / b, \Delta_{0}, \Delta_{i}\right) \geqslant-|z| \max (m, \alpha) \sqrt{ }\left\{V\left(\mathbf{X}_{0}, \mathbf{X}_{i} / b, \Delta_{0}, \Delta_{i}\right)\right\}
$$

ACKNOWLEDGEMENTS

The author would like to thank the editor and referees for many suggestions which improved the presentation of this paper. This work was supported by the National Science Council of Taiwan under contract number NSC86-2115-M-008-018.

\section{REFERENCES}

1. Hochberg, Y. and Tamhane, A. C. Multiple Comparison Procedures, Wiley, New York, 1987.

2. Chakraborti, S. and Desu, M. M. 'Linear rank tests for comparing treatments with a control when data are subject to unequal patterns of censorship', Statistica Neerlandica, 45, 227-254 (1991).

3. Slepian, D. The one-sided barrier problem for Gaussan noise', Bell System Technical Journal, 41, 463-501 (1962).

4. Gehan, E. A. 'A generalized Wilcoxon test for comparing arbitrarily singly-censored samples', Biometrika, 52, 203-223 (1965).

5. Chen, Y. I. 'A generalized Steel's procedure for comparing several treatments with a control under random right-censorship', Communications in Statistics - Simulation and Computation, 23, 1-16 (1994).

6. Steel, R. G. D. 'A multiple comparison rank sum test: treatments versus control', Biometrics, 15, 560-572 (1959). 
7. Mantel, N. 'Evaluation of survival data and two new rank order statistics arising in its consideration', Cancer Chemotherapy Reports, 50, 163-170 (1966).

8. Fleming, T. R. and Harrington, D. P. Counting Process nad Survival Analysis, Wiley, New York, 1991.

9. Chen, Y. I. 'Simple-tree weighted logrank tests for right-censored data', Annals of the Institute of Statistical Mathematics, 50, 311-324 (1998).

10. Wei, L. J. and Gail, M. H. 'Nonparametric estimation for a scale-change with censored observations', Journal of the American Statistical Association, 78, 382-388 (1983).

11. Marcus, R., Peritz, E. and Gabriel, K. R. 'One closed testing procedures with special reference to ordered analysis of variance', Biometrika, 63, 655-660 (1976).

12. Peto, R. and Peto, J. 'Asymptotically efficient rank invariant test procedures (with discussion)', Journal of the Royal Statistical Society, Series A, 135, 185-206 (1972).

13. Prentice, R. L. 'Linear rank tests with right censored data', Biometrika, 65, 165-179 (1978).

14. Gately, M. K. 'Interleukin-12: a recently discovered cytokine with potential for enhancing cell-mediated immune responses to tumours', Cancer Investigations, 11, 500-506 (1993).

15. Maas, R. A., Dullens, H. F. and Den Otter, W. 'Interleukin-2 in cancer treatment: disappointing or (still) promising? A review', Cancer Immunology Immunotherapy, 36, 141-148 (1993).

16. Rossi, A. R., Pericle, F., Rashleigh, S., Janier, J. and Djeu, J. Y. 'Lysis of neuroblastoma cell lines by human natural killer cells activated by interleukin-2 and interleukin-12, Blood, 83, 323-328 (1994).

17. Wigginton, J. M., Komschlies, K. L., Back, T. C., Franco, J. L., Brunda, M. J. and Wiltrout, R. H. 'Administration of interleukin 12 with pulse interleukin 2 and the rapid and complete eradication of murine renal carcinoma', Journal of the National Cancer Institute, 88, 38-43 (1996).

18. Kaplan, E. L. and Meier, P. 'Nonparametric estimator from incomplete observations', Journal of the American Statistical Association, 53, 457-481 (1958).

19. Schervish, M. J. 'Multivariate Normal Probabilities with error bound', Applied Statistics, 33, 81-94 (1984).

20. Gupta, S. S. 'Probability integrals of multivariate normal and multivariate t', Annals of Mathematical Statistics, 34, 792-828 (1963).

21. Dunnett C. W. and Tamhane, A. C. 'Step-down multiple tests for comparing treatments with a control in unbalanced one-way layouts', Statistics in Medicine, 10, 939-947 (1991).

22. Anderson, P. K., Borgan, O., Gill, R. D. and Keiding, N. Statistical Models Based on Counting Processes, Springer-Verlag, New York, 1993.

23. Dunnett, C. W. 'New tables for multiple comparisons with a control', Biometrics, 20, 482-491 (1964). 